\title{
Post-operative Epiphora Following the Transcutaneous Medial Canthal Incision
}

Mark A Prendes, MD ${ }^{1,2,3}$; John Mittel, BS ${ }^{1}$; Peter J Timoney, MD $^{4}$; Christopher J Compton,

$\mathrm{MD}^{1}$; Jeremy D Clark, MD ${ }^{1}$; William R Nunery, MD, FACS ${ }^{1,2}$; Jonathan Y Ting, MD ${ }^{5}$; Taha Z.

Shipchandler, MD FACS ${ }^{5}$; H.B. Harold Lee, MD, FACS ${ }^{2}$

1 Department of Ophthalmology and Visual Sciences, University of Louisville, Louisville,

Kentucky, U.S.A.

2 Oculofacial Plastic and Orbital Surgery, Indianapolis, Indiana, U.S.A

3 University Hospitals Eye Institute, Cleveland, Ohio, U.S.A.

4 Department of Ophthalmology, University of Kentucky College of Medicine, Lexington,

Kentucky, U.S.A.

5 Department of Otolaryngology—Head \& Neck Surgery, Indiana University School of

Medicine, Indianapolis, Indiana, U.S.A.

This work was not presented at any meetings or submitted elsewhere for publication

This research was funded in part by Research to Prevent Blindness, New York, NY.

Declarations of interest: none

Corresponding Author:

Mark A. Prendes, MD

University Hospitals Eye Institute

11100 Euclid Ave, Lksd 4107

Cleveland, $\mathrm{OH} 44106$

Phone: 216-844-7029

Email:markprendes@gmail.com

Running Head: Iatrogenic epiphora

This is the author's manuscript of the article published in final edited form as:

Prendes, M. A., Mittel, J., Timoney, P. J., Compton, C. J., Clark, J. D., Nunery, W. R., ... Lee, H. B. H. (2019). Post-operative epiphora following the transcutaneous medial canthal incision. American Journal of Otolaryngology, 40(4), 564-566. 
Key words: Medial canthal incision, Transcutaneous, Medial orbit, Orbitotomy, Transcaruncular, medial canthus, epiphora 
Precis: The transcutaneous medial canthal incision is an important tool to gain access to the medial orbit in select patients and appropriate surgical technique portends a low risk of postoperative complications. 


\begin{abstract}
:
Purpose: The safety profile of the transcutaneous medial canthal incision for access to the medial orbit is assessed with a focus on the risk of post-operative iatrogenic epiphora. Methods: A retrospective chart review of patients undergoing medial orbitotomy via the transcutaneous medial canthal incision was performed. Patients with a minimum of 3 months of follow-up were included and post-operative complications were assessed and characterized. Results: Onehundred-fifty patients were included in the study. A total of 4 complications were identified, including one each of the following: nasolacrimal duct obstruction, hypertrophic scar, suture granuloma and soft tissue infection. Only the nasolacrimal duct obstruction required surgical intervention. Discussion: Access to the medial orbit has been achieved through a variety of approaches, each with their own benefits and risk profile. The transcaruncular approach has increased in usage as a means to avoid a visible cutaneous scar and decrease the risk of iatrogenic epiphora, however, there are specific patients who may have relative contraindications to this approach. The current study demonstrates the low risk profile of the transcutaneous medial canthal incision, specifically the minimal risk of iatrogenic damage to the nasolacrimal outflow system. This approach is another useful tool which orbit surgeons should be familiar with to offer as an option to patients requiring medial orbitotomy.
\end{abstract}


Introduction:

The transcutaneous medial canthal tendon incision has previously been described as a low-risk, highly effective way to gain access to the medial orbital wall. [1-3] This incision may be utilized for multiple procedures including external dacryocystorhinostomy, medial orbital wall fracture repair, external ethmoidectomy for orbital decompression, open repair of nasal bone fractures and medial orbitotomy for incisional or excisional biopsy. As previously described, this technique requires lateral retraction of the lacrimal sac and elevation of the medial canthal tendon, including the posterior limb, known as Horner's muscle. [1] Horner's muscle is an important component of the tear pump mechanism. Anecdotally, a common transient complaint of epiphora postoperatively has been noted, which resolves in the majority of patients within one month. There are multiple reasons for these patients to have symptomatic post-operative epiphora, all of which should resolve within a few weeks. The current investigation sought to answer whether or not a subset of patients experience permanent epiphora postoperatively, and review the identified etiology and treatment. A complete understanding of the risks inherent to this surgical approach can allow surgeons to better counsel patients preoperatively and better manage complications postoperatively. Furthermore, understanding the low risk profile and surgical technique of this approach to the medial orbit provides surgeons with another tool that can be utilized following appropriate patient selection.

Methods: 
Institutional Review Board approval was received from the University of Louisville for the project and the study was compliant with the Declaration of Helsinki. The study was HIPAAcompliant, with protection of individually identifiable health information. A retrospective chart review was performed on all patients undergoing a medial orbitotomy at the University of Louisville between January 1, 2008 and January 1, 2017. The following CPT codes were used for the search: 31205, 67412, 67400, 67405, 67413, 21407, 21346-8. Patients who had undergone one of these procedures through use of a transcutaneous medial canthal tendon incision and who had a minimum of 3 months follow-up were included in the study. Patients were excluded if they did not have a minimum of 3 months follow-up, if they had eyelid retraction which was not addressed at the time of surgery, if they had undergone prior lacrimal surgery, if they had received previous radioactive iodine therapy or if they were being subsequently evaluated for fractures involving the nasolacrimal duct. Chart review was performed to obtain patient demographics, preoperative diagnosis, procedure, laterality, intraoperative complications, presence or absence of post-operative epiphora at post-operative week one and post-operative month one to three visits, whether or not irrigation of the lacrimal system was performed, if post-operative epiphora was present then the etiology was sought, any secondary procedures performed, presence of any other complications from the incision, and the length of follow-up.

Results:

After exclusion criteria, 150 patients were included in the study. The average age was 47.6 years +/- 19.1 (Median 47, range 4-84 years). Fifty-one (51) males were included and 99 females, likely reflecting the higher propensity of Graves' orbitopathy in women, as a large number of the 
procedures were orbital decompressions. The average follow-up period post-operatively was 14.5 months. Eighty-two (82) patients underwent orbital decompression surgery with use of the transcutaneous medial canthal incision for external ethmoidectomy. Sixty-one (61) patients underwent fracture repair with use of the incision to access a large medial orbital wall fracture. Five (5) patients underwent the procedure for drainage of an orbital or subperiosteal abscess. Two (2) patients had a biopsy of a medial orbital mass through the incision. Surgery was performed on 71 Right orbits and 79 left orbits.

Seventy-nine (79) patients $(52.7 \%)$ reported epiphora at their post-operative week 1 visit. This number decreased to 12 patients $(8 \%)$ with epiphora reported at their post-operative month 1-3 appointment. Therefore, $84.8 \%$ of patients with epiphora at post-operative week 1 will have resolution within the first 1 to 3 months after surgery. Of the patients with epiphora at postoperative week $1,15.2 \%$ will therefore have some persistence of epiphora greater than one month after surgery.

Of the 12 patients with epiphora at the post-operative month 1-3 visit, all of them had irrigation of their lacrimal system performed in the office for diagnostic evaluation. $11(91.7 \%)$ of the patients had patent systems on irrigation, while $1(8.3 \%)$ of the patients irrigated had evidence of nasolacrimal duct obstruction. This patient had undergone bilateral orbital decompressions with orbital floor and medial wall decompressions. She reported left-sided epiphora at post-operative month one and underwent bilateral irrigation with a patent system on the right and evidence of nasolacrimal duct obstruction on the left. Of note, during the subsequent dacryocystorhinostomy, intraoperative findings demonstrated a normal appearing lacrimal sac without evidence of prior 
iatrogenic trauma. The other patients with epiphora at post-operative months 1-3 who had patent systems were diagnosed with epiphora secondary to: Dry eye syndrome with reflex tearing (7 patients) and Conjunctivochalasis or punctal stenosis (4 patients). Each of these patients had resolution of epiphora following treatment with lubrication and lid hygiene, or conjunctivoplasty and punctoplasty, respectively.

Assessment for other complications from the medial canthal incision revealed that there were no complications in 146 patients (97.3\%). One (1) patient had a suture granuloma which resolved without intervention. One (1) patient had persistent inflammation around the incision which resolved after treatment with oral antibiotics for suspicion of soft tissue infection. One patient developed a mild hypertrophic scar without webbing but elected not to have further intervention.

\section{Discussion:}

Surgical access to the medial orbit requires thorough knowledge of orbital anatomy and meticulous technique to avoid injury to multiple important ocular and ocular adnexal structures. Many surgeons have become familiar with the transcaruncular approach to the medial orbit and have abandoned transcutaneous approaches. [4] While the transcaruncular incision has the benefit of avoiding a skin incision, comfort with the transcutaneous incision is important so that each case can be addressed individually depending on specific patient needs. For instance, in patients with glaucoma, avoidance of manipulation of the conjunctiva is important. Prior studies have reported complications of the transcaruncular incision including cicatrization of the conjunctiva and massive sunconjunctival hemorrhage. [5,6] Both of these complications are undesirable in any patient, but could be particularly detrimental in patients with glaucoma where 
they could lead to premature failure of trabeculectomy or may prevent its future feasibility. Previous authors have recommended caution in use of a transcaruncular approach in patients with ocular surface disorders including significant dry eye, inflammatory or cicatricial conjunctival disease. [5] The transcutaneous medial canthal incision has also been shown to be beneficial in patients with nasoorbitoethmoid fractures who may benefit from the excellent exposure to the medial orbital wall for repair as well as direct exposure of the nasal bones for mini-plating. [2]

Complication rates have been low in the reported literature on transcaruncular incisions. A previous study by Graham et al reported a 1.7\% complication rate for transcaruncular incision with the single complication (1/58 orbits) being a patient with medial fornix scarring causing restrictive strabismus and symptomatic diplopia which required a subsequent revision surgery. [6] Additional studies have reported post-operative inferior oblique underaction, hypertrophic scarring of the caruncle, and partial canalicular obstruction. [5,7] A more recent review of literature on the transcaruncular approach found that the average overall complication rate for the eleven studies reviewed was $2.6 \%$. [8] This overall complication rate was similar to our current study which demonstrated a surgical complication rate of $2.7 \%(4 / 150)$. The transcutaneous approach did not lead to any injury to the extraocular muscles or scarring of the ocular surface and could therefore arguably have a less serious risk profile than the transcaruncular approach.

It is unclear if the patient in the current study found to have post-operative nasolacrimal duct obstruction had the obstruction prior to surgical intervention, or if the surgery itself caused the obstruction. This patient had complaints of epiphora upon initial presentation, but the epiphora 
was suspected to be related to reflex epiphora secondary to corneal exposure so irrigation of the lacrimal outflow system was not performed. When the epiphora did not resolve by 1-3 months after surgery, only then was irrigation performed which revealed the obstruction. During subsequent dacryocystorhinostomy there was no evidence of prior iatrogenic injury to the lacrimal sac and the obstruction was more consistent with obstruction of the intraosseous nasolacrimal duct.

In summary, due to the aforementioned benefits, surgeons should be familiar with both transcutaneous and transcaruncular approaches to the medial orbit, including the risks and benefits of each, so that patients can be individually addressed with an approach that is optimal to manage their pathology. The current study documents the low risk profile of the transcutaneous medial canthal incision for access to the medial orbital wall. It specifically reveals that while elevation of the posterior limb of the medial canthal tendon, or Horner's muscle, is performed, this does not lead to permanent functional epiphora. Also, lateral retraction of the lacrimal sac has minimal risk of causing damage to the nasolacrimal drainage system. The benefits of the incision include wider surgical exposure while minimizing risk of injury to intraorbital anatomy. Thorough understanding of the anatomic relationships of the anterior medial orbit and meticulous surgical technique allow for use of this safe and beneficial approach to access the medial orbit. 
References:

[1].Timoney PJ, Sokol JA, Hauck MJ, Lee HB, Nunery WR. Transcutaneous Medial Canthal Tendon Incision to the Medial Orbit. Ophthal Plast Reconstr Surg. 28(2): 2012.

[2].Nunery WR, Tao JP. Medial canthal open nasal fracture repair. Ophthal Plast Reconstr Surg. 2008 Jul-Aug;24(4):276-9.

[3].Nunery WR, Tao JP, Johl S. Nylon foil "wraparound" repair of combined orbital floor and medial wall fractures. Ophthal Plast Reconstr Surg 2008;24:271-5.

[4].Kim SJ, Lew H, Chung SH, et al. Repair of medial orbital wall fracture: transcaruncular approach. Orbit 2005;24:1-9.

[5].Malhotra R, Saleh G, de Sousa JL, Sneddon K, Selva D. The Transcaruncular Approach to Orbital Fracture Repair: Ophthalmic Sequelae. J of Craniofac Surg. 18(2): March 2007. 
[6]. Graham SM, Thomas RD, Carter KD, Nerad JA. The Transcaruncular Approach to the Medial Orbital Wall. The Laryngoscope. 112: June 2002.

[7]. Garcia GH, Goldberg RA, Shorr N. The transcaruncular approach in repair of orbital fractures: a retrospective study. J Craniomaxillofac Trauma 1998;4:7-12.

[8].Choi M, Flores RL. Medial Orbital Wall Fractures and the Transcaruncular Approach. J of Craniofac Surg. 23(3):May 2012.

[9]. Rootman J, Stewart B, Goldberg RA. Orbital surgery: A conceptual approach, Philadelphia, Lippincott-Raven, 1995;38-42. 